\title{
Fortalecimiento de la gestión empresarial en las pequeñas y medianas empresas en Ecuador, a partir del uso de herramientas cualitativas y cuantitativas
}

\section{Strengthening of business management in small and medium enterprises in Ecuador, based on the use of qualitative and quantitative tools}

Yailí Pérez Guerra

Universidad Internacional del Ecuador, Ecuador

Autor para correspondencia: yaperezgu@internacional.edu.ec

Fecha de recepción: 30 de Agosto de 2016 - Fecha de aceptación: 25 de Octubre de 2016

Resumen: La globalización ha colocado a las organizaciones en una difícil situación, forzando una evolución en los modelos y estándares de gestión empresarial. A esta realidad no quedan exceptas las pequeñas y medianas empresas (pymes), las cuales deben alcanzar una gestión adecuada y coherente, independientemente de su tamaño como entidad empresarial. Las pymes en Ecuador tienen una fuerte importancia para la economía nacional, por el número de empleos que representan y por su contribución a la derrama financiera. Es conocido que un elemento determinante en los resultados empresariales son los procesos decisionales, los cuales pueden fortalecerse a partir de su apoyo en herramientas cualitativas y cuantitativas, lo que minimiza los riesgos y niveles de incertidumbre, y permite que sean ejecutados de forma rápida y eficiente; conllevando a mejores resultados en las organizaciones. Ante esta necesidad, el presente trabajo se ha trazado como objetivo analizar un conjunto de herramientas cualitativas y cuantitativas que sirvan de apoyo al proceso de toma de decisiones y por ende contribuyan al desarrollo empresarial en las pymes. Como resultado de la investigación se ofrece una panorámica de este sector empresarial en Ecuador y se recomiendan algunas herramientas y técnicas que pudieran ser utilizadas con vistas a obtener mejores resultados económicos.

Palabras claves: negocios; PYMES; herramientas cualitativas; herramientas cuantitativas Abstract: Globalization has placed organizations under a difficult situation, pushing and evolving models and standards of enterprise management. This reality is no exception to small and medium enterprises (SMEs) which must reach a coherent and adequate management, independent of the organization size. SMEs in Ecuador have a strong importance for the national economy because of the number of job positions they offer and because of their financial contribution. It is known that a determinant element in the enterprise results are decision processes which can become stronger if supported using qualitative and quantitative tools which minimize risks and uncertainty levels and permits execution to be fast and efficient; resulting in better outcomes for organizations. In front of this need the objective of the following work is to analyze a group of quantitative and qualitative tools that support the process of decision making and that contribute to the enterprise development on SMEs. As result of this research a panoramic view of Ecuador's enterprise sector is offered and some tools and techniques are recommended that could be used with projections to obtain better economic results.

Key words: business; SME; qualitative tools; quantitative tools 
En un escenario de alta volatilidad internacional como el que nos encontramos inmersos, son las pequeñas y medianas empresas quienes pueden generar los paliativos necesarios para que en caso de que existan shocks internacionales, estos nos afecten en menor medida o no nos afecten. (Secretaría Nacional de Planificación y Desarrollo, 2012)

\section{Introducción}

Las pymes vislumbran grandes oportunidades de crecimiento y desarrollo económico para cualquier nación, por lo que resulta importante mejorar y fortalecer su gestión para que paulatinamente puedan transformarse en organizaciones altamente eficientes y eficaces. Esto a su vez también se traduce en nuevas fuentes de empleo y generación de divisas para la sociedad y el país.

Ante la inestabilidad y variabilidad de los sistemas económicos actuales cobran cada vez mayor fuerza los procesos de toma de decisiones y la eficacia de sus resultados, los cuales repercuten directamente en los resultados a corto, mediano y largo plazo de cualquier entidad económica. Estas condiciones trascienden a todos los marcos de las organizaciones, llegando a convertirse en una habilidad indispensable en las actividades de dirección el dominio y manejo de técnicas y herramientas para lograr la efectividad de los proceso decisionales.

Toda decisión trae asociado implícitamente un nivel de incertidumbre, una forma de minimizarlo es cambiando los paradigmas decisionales de "un enfoque empírico a un enfoque científicamente argumentado", orientado a buscar soluciones que reduzcan los costos totales, y mejores la calidad de los productos y servicios. (Garza Ríos, González Sánchez, Pérez Vergara, Martínez Delgado, \& Sanler Cruz, 2012)

Las premisas anteriores han llevado a plantearse las siguientes interrogantes: a) ¿Cuáles son las condiciones actuales de las pymes en Ecuador?; b) ¿Se puede contribuir al desarrollo empresarial en pymes a partir de la utilización de técnicas cualitativas y cuantitativas como sustento a los procesos decisionales?; c) ¿Qué herramientas pueden ser utilizadas?; d) ¿Qué efectos pudieran tener en la gestión de las pymes la aplicación de herramientas cualitativas y cuantitativas? objetivos.

Para darle respuesta a las anteriores preguntas la investigación se han trazado los siguientes

\section{Objetivo general}

Analizar los procesos decisionales en las organizaciones y las herramientas cuantitativas y cualitativas que pueden mejorar su efectividad en las pequeñas y medianas empresas. Dicho objetivo se le dará cumplimiento paulatinamente a partir de los siguientes

\section{Objetivos específicos:}

1. Realizar un análisis de las pequeñas y medianas empresas, teniendo en cuenta su importancia y estado actual en Ecuador. 
2. Desarrollar un estudio del estado del arte y la práctica de los procesos decisionales y las principales herramientas cualitativas y cuantitativas que pueden ser utilizados como apoyo de estos.

3. Proponer un conjunto de herramientas cualitativas y cuantitativas que sustenten la toma de decisiones en las pymes y propicien a mejora en su gestión.

Como resultado de la investigación se espera llegar a tener una idea clara de la situación actual del sector de las pymes en Ecuador, cuáles son las necesidades y limitantes para su desarrollo y proponer un conjunto de herramientas que puedan ayudarles a mejorar.

\section{Resultados}

\section{Empresa. Pequeñas y medianas empresas (pymes)}

La definición de empresa ha sido ampliamente abordada y analizada; el desarrollo de la industria y la sociedad fueron determinando la forma en que los teóricos la percibían ampliando su alcance y fuerza. Paulatinamente fue evolucionando de un argumento meramente económico productivo hasta esferas mucho más amplias que involucran la psicología del hombre, su responsabilidad con la sociedad y el medio ambiente. No es objeto del presente trabajo ahondar en la definición de empresa, pero su conocimiento si resulta una premisa importante para el desarrollo de la investigación. Para ello se debe analizar inicialmente ¿Qué es una empresa?

Extrayéndose los elementos comunes de las definiciones dadas por (Andersen , 1999); (Romero R. , 1999); (García Del Junco \& Casanueva Rocha, 2001); (Pérez Gorostegui, 1994) y (Bueno Campos, 2007) y agregándoles los aportes específicos de cada autor ser puede proponer a modo de resumen que: una empresa es una unidad económica conformada por bienes materiales, técnicos, financieros y humanos, donde su integración y funcionamiento como sistema conlleva a la concreción de sus objetivos; busca obtener beneficios financieros a partir de la coordinación de factores de producción, comercialización y marketing tanto para productos como servicios, y se orientan a la satisfacción de sus clientes.

Los anteriores son los elementos más relevantes, no obstante (Méndez Morales , 1996) realizó un estudio donde destaca las características con que cualquier empresa contará, de las cuales sea desean destacar cuatro en especial, véase la Figura 1. 


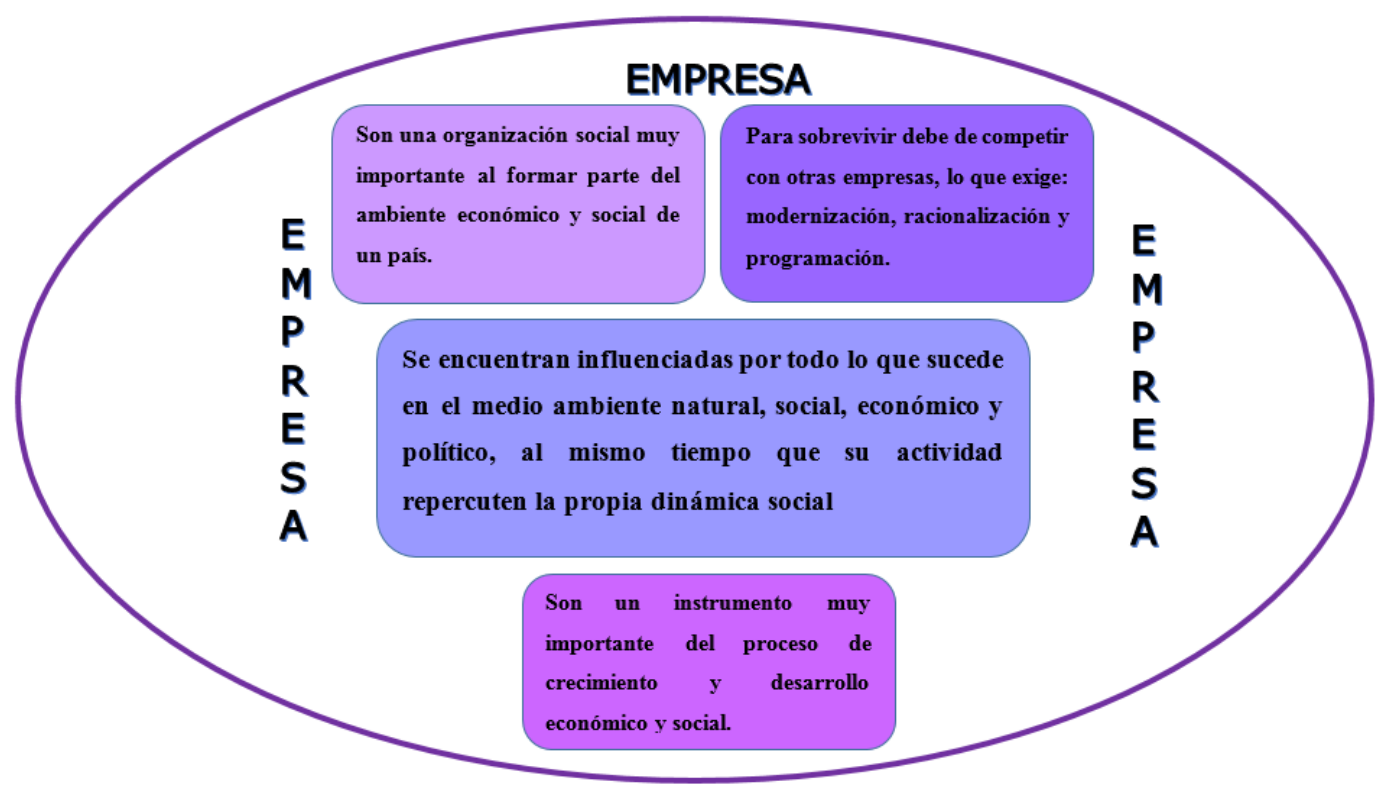

Figura 1. Características de las empresas Adaptado de: (Méndez Morales , 1996)

La perspectiva de este autor resulta muy interesante; no la ve como una unidad aislada o independiente, si no que la enmarca en un sistema del cual es parte, el cual repercute sobre ella y ella determina también su desarrollo.

Comprender que las empresas son parte de un engranaje superior, es una premisa en la presente investigación. En la medida que estas crezcan, se desarrollen y mejoren repercutirán mediante una correspondencia directa en la economía nacional y la sociedad, las cuales paralelamente se desarrollarán, crecerán y mejorarán.

Las definiciones estudiadas de "empresa" son generalizadoras, pero no todas son iguales, pueden segmentarse de acuerdo a la actividad que realizan, su tamaño, forma de propiedad, entre otras variables, lo que las particulariza y facilita su análisis. De acuerdo a su tamaño se clasifican en: grandes, medianas y pequeñas empresas. Las pequeñas y medianas empresas suelen tratarse bajo una óptica común y se les denomina por el acrónimo: pyme. (Fundéu-BBVA, 2011)

No existe un consenso a la hora de definir cuando una empresa entra en esta categoría, por lo general cada país tiene un estándar diferente para su clasificación y suele sustentarse en los valores de sus ingresos y facturación anual. (Universidad San Martín de Porres, 2012)

Independientemente del país donde estén establecidas las pymes, los autores resaltan numerosas ventajas, así como desventajas, en su funcionamiento, por su tamaño y características propias, las cuales pueden observarse en la Figura 2. 


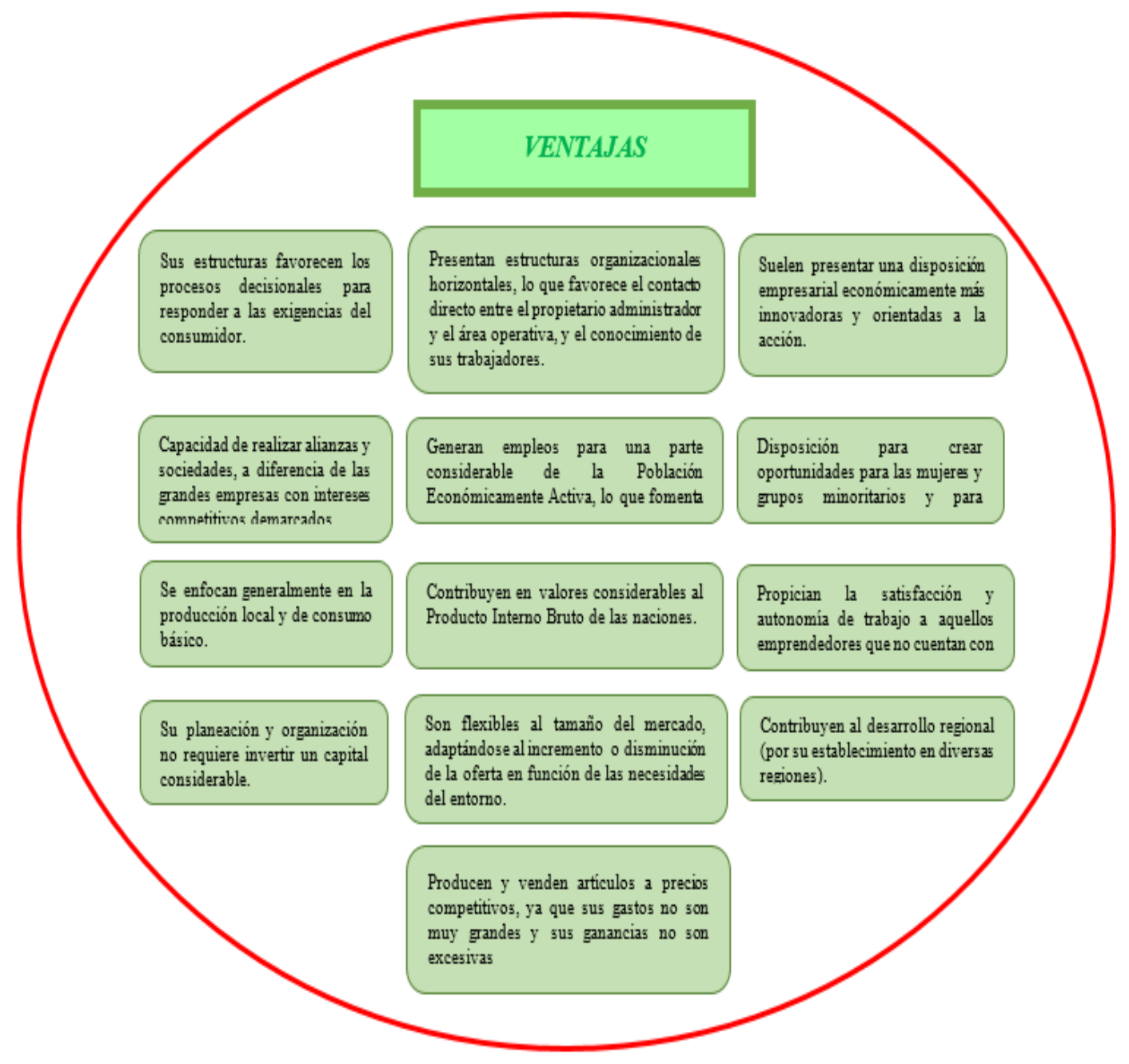




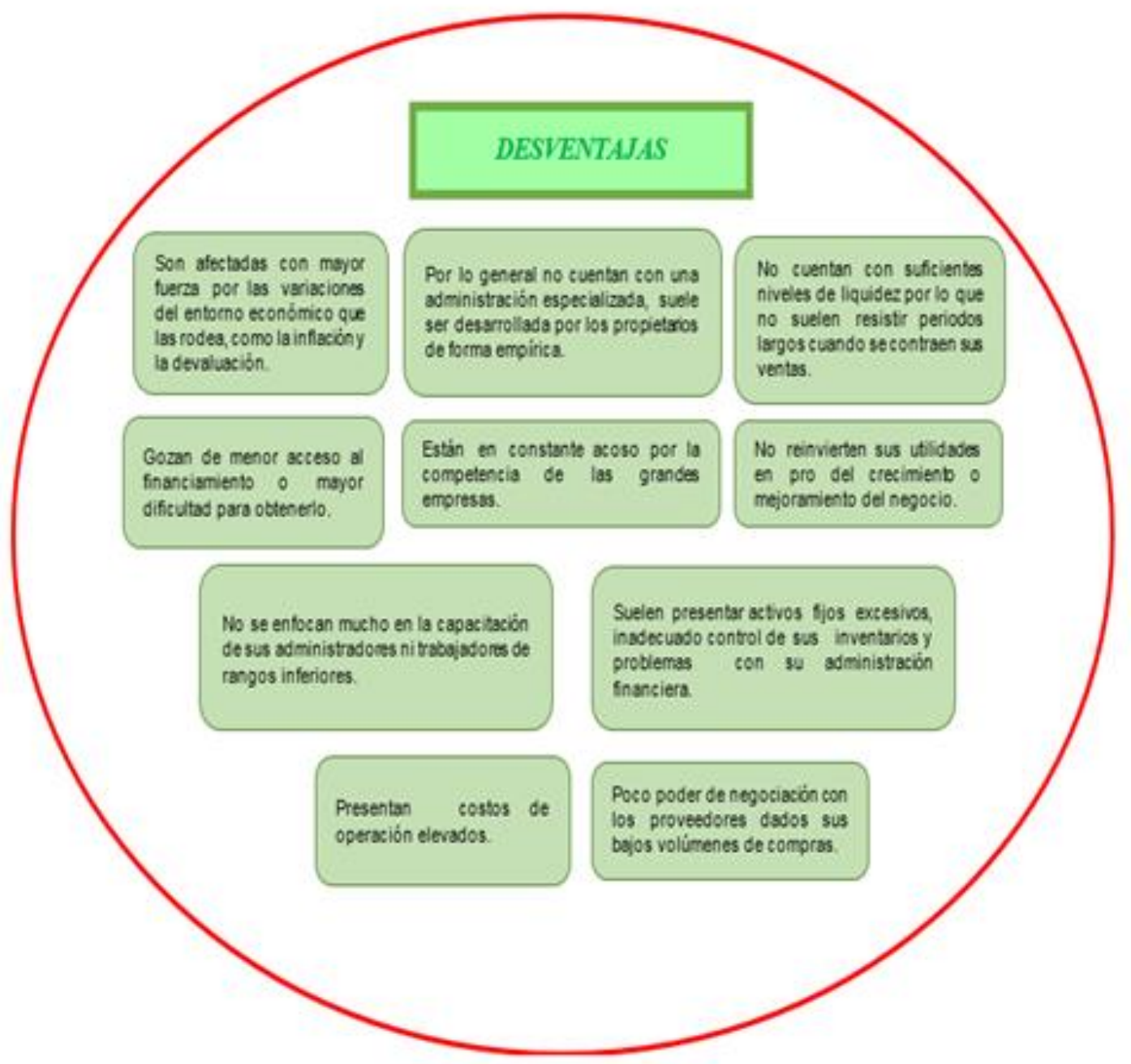

Figura 2. Ventajas y desventajas en el funcionamiento de las pymes.

Adaptado de: (Albanese \& Boland, 2004); (Universidad San Martín de Porres, 2012); (Lemes Batista \& Machado

Hernández, 2007); (Amaro Zárate, 2003) (Rodríguez Valencia, 2002) y (Amaro Zárate, 2003)

Muy aparte de las ventajas o desventajas que presentan, se debe destacar su importancia. Las pymes son una alternativa para el desarrollo, favoreciendo el tránsito a la industrialización, por lo que resulta un sustento para el avance económico del país. Ante las dificultades que entorpecen su surgimiento, crecimiento y mantenimiento hay que buscar alternativas de solución.

\section{Pequeñas y medianas empresas en Ecuador}

De acuerdo a (Instituto Nacional de Estadísticas y Censos, 2014) una empresa “ es un agente económico (persona natural o sociedad) con autonomía para adoptar decisiones financieras y de inversión y con autoridad y responsabilidad para asignar recursos a la producción de bienes y servicios y que puede realizar una o varias actividades productivas." Su estratificación, de acuerdo a su tamaño está definida de acuerdo con el volumen de ventas anual (V) y el número de personas ocupadas $(\mathrm{P})$, en la siguiente figura se puede ver los límites para clasificarlas. 


\begin{tabular}{lll}
\hline Clasificación de las empresas & Volúmenes de ventas anuales & Personal ocupado \\
\hline Micro empresa & Menor a 100.000 & $1 \mathrm{~A} 9$ \\
\hline Pequeña empresa & De 100.001 a 1'000.000 & 10 A 49 \\
Mediana empresa "A" & De 1'000.001 a 2'000.000 & 50 A 99 \\
\hline Mediana empresa "B" & De 2'000.001 a 5'000.000 & 100 A 199 \\
Grande empresa & De 5'000.001 en adelante & 200 en adelante \\
\hline
\end{tabular}

Figura 3. Parámetros para clasificar las empresas en Ecuador.

Tomado de: (Instituto Nacional de Estadísticas y Censos, 2014) ${ }^{1}$

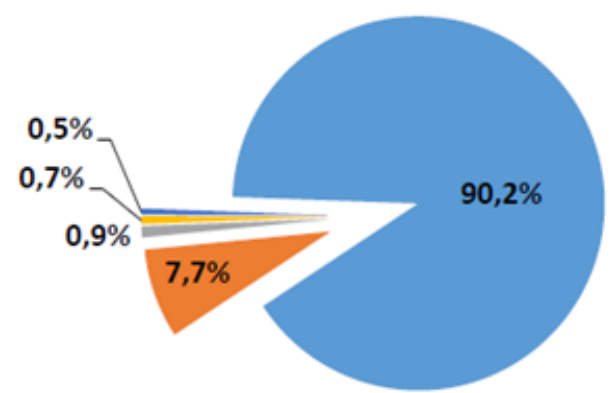

\author{
Microempresa \\ - Pequeña empresa \\ = Mediana empresa "A" \\ Mediana empresa "B" \\ Grande empresa
}

Figura 4. Desglose del sector empresarial ecuatoriano de acuerdo a su tamaño. Tomado de: (Instituto Nacional de Estadísticas y Censos, 2014)

Del total de empresas registradas a nivel nacional solo el 0,5\% pertenecen a la categoría de "Grande empresa", lo cual puede observarse en la Figura 4. Esta situación resalta una vez más la importancia de trabajar en la mejora de la gestión en las pequeñas y medianas empresas, dado su fuerte repercusión en la economía nacional, siendo las pymes la célula básica del tejido productivo nacional y elemento indispensable para el crecimiento económico.

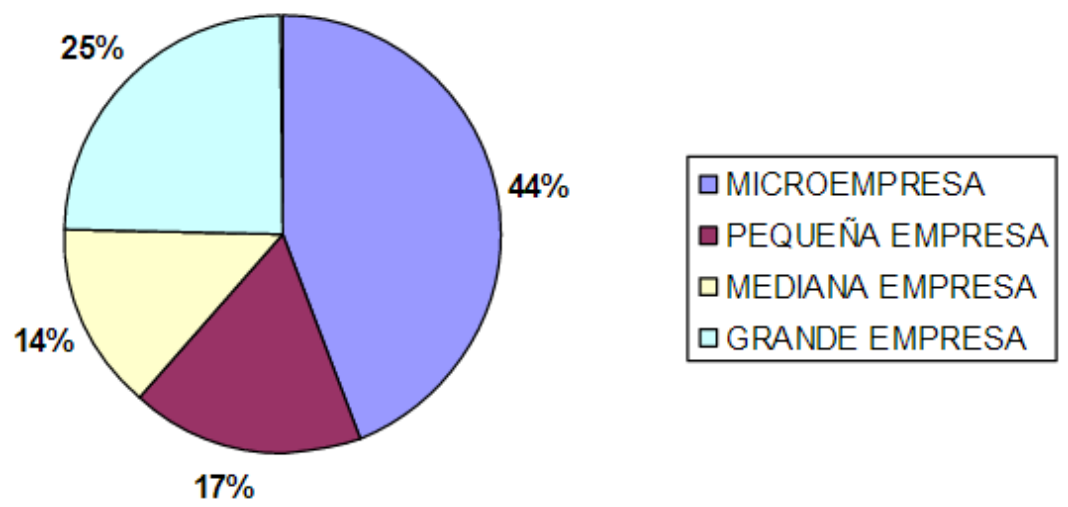

Figura 5. Aporte a la generación de empleo en Ecuador.

Tomado de: (INEC, 2010)

Del total de personas con una relación laboral formal las pymes generan empleo al $75 \%$ de esta categoría social.

\footnotetext{
${ }^{1}$ Nota: clasificación emitida por la Comunidad Andina de Naciones (CAN). Es importante señalar que el 1er criterio para determinar el tamaño de una empresa son las ventas y en 2 do lugar el personal ocupado.
} 

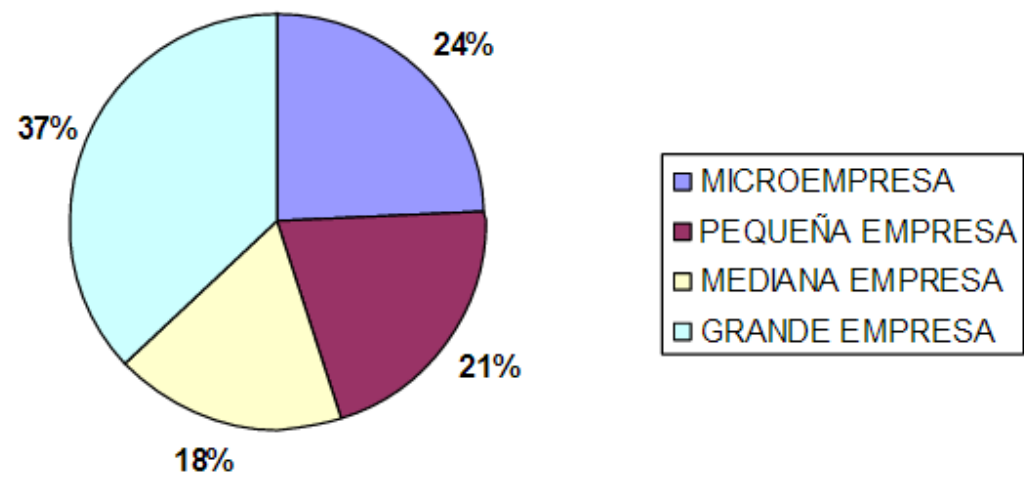

Figura 6. Aporte a la generación de ingresos por ventas.

Tomado de: (INEC, 2010)

El 63\% de los ingresos en la población son generados por las ventas en las micro, pequeñas y medianas empresas; un valor notablemente superior a lo que perciben como resultado del funcionamiento de las grandes empresas.
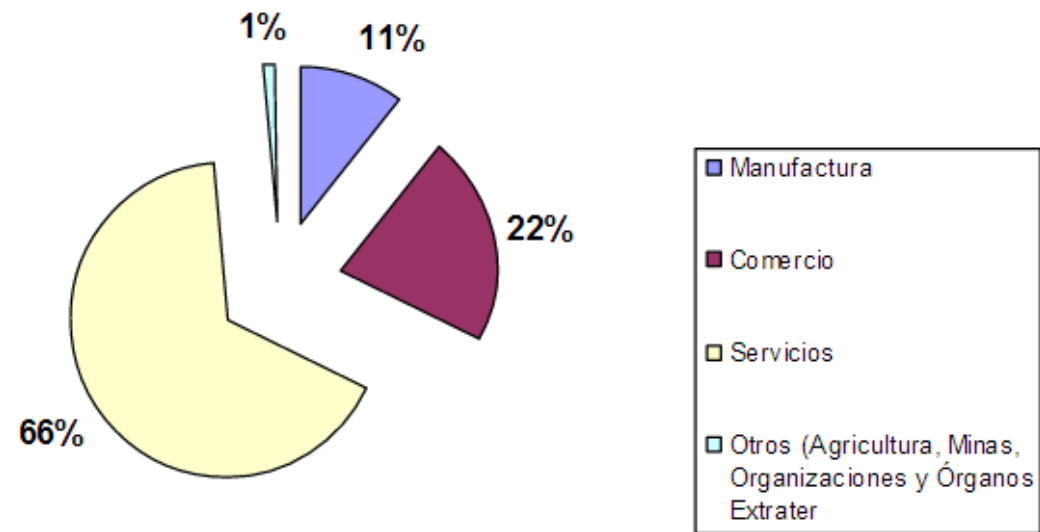

Figura 7. Pequeñas y medianas empresas por sector.

Tomado de: (INEC, 2010)

Al analizarse los sectores donde más se han desarrollado las pymes se destacan los servicios en primer lugar y el comercio en segundo, con un $88 \%$ del total. Debería prestarse especial atención al resto de los sectores para apoyar su crecimiento, analizando primeramente las causales de sus bajos niveles de participación en la economía nacional.

En la siguiente figura se muestran dentro del área de los servicios las diez principales actividades en que se desempeñan las pymes. 


\begin{tabular}{|c|c|c|}
\hline & Establecimientos & $\%$ \\
\hline $\begin{array}{l}\text { Venta al por menor en comercios no especializados } \\
\text { con predominio de la venta de alimentos, bebidas y } \\
\text { tabaco }\end{array}$ & 87,244 & $17.4 \%$ \\
\hline $\begin{array}{l}\text { Actividades de restaurantes y de servicio móvil de } \\
\text { comidas. }\end{array}$ & 44,722 & $8.9 \%$ \\
\hline $\begin{array}{l}\text { Venta al por menor de prendas de vestir, calzado y } \\
\text { artículos }\end{array}$ & 25,432 & $5.1 \%$ \\
\hline $\begin{array}{l}\text { Mantenimiento y reparación de vehículos } \\
\text { automotores. }\end{array}$ & 20,437 & $4.1 \%$ \\
\hline Otras actividades de telecomunicaciones. & 17,528 & $3.5 \%$ \\
\hline $\begin{array}{l}\text { Venta al por menor de alimentos, bebidas y tabaco } \\
\text { en puestos }\end{array}$ & 15,627 & $3.1 \%$ \\
\hline $\begin{array}{l}\text { Otras actividades de venta al por menor en } \\
\text { comercios no especializado }\end{array}$ & 15,474 & $3.1 \%$ \\
\hline $\begin{array}{l}\text { Venta al por menor de alimentos en comercios } \\
\text { especializados. }\end{array}$ & 14,634 & $2.9 \%$ \\
\hline $\begin{array}{l}\text { Actividades de peluquería y otros tratamientos de } \\
\text { belleza. }\end{array}$ & 14,426 & $2.9 \%$ \\
\hline $\begin{array}{l}\text { Venta al por menor de productos farmacéuticos y } \\
\text { medicinales, }\end{array}$ & 10,884 & $2.2 \%$ \\
\hline Los 229 sectores restantes & 233,809 & $46.7 \%$ \\
\hline
\end{tabular}

Figura 8. Por número de establecimientos de acuerdo a las diez actividades económicas principales a nivel nacional. Tomado de: (Araque J., 2012)

Del análisis anterior se puede concluir que las pymes juegan un fuerte rol como parte del tejido empresarial, con repercusiones sociales evidentes, por lo cual debe dársele un apoyo determinante por parte del Gobierno Nacional para lograr una nación con igualdad de oportunidades, que minimice las desigualdades de gestión, operación y mercado e impulse el cambio de la matriz productiva. EI Ministerio de Industrias y Productividad en su visión sobre la política productiva nacional busca una gestión incluyente, articulada y participativa, que tiene como objetivo, propiciar el crecimiento del sector industrial y fundamentalmente las micro, pequeñas y medianas empresas. (González Jaramillo, 2013)

El desarrollo de este segmento de la economía ecuatoriana contribuiría a la mejora de la matriz productiva en dos aspectos fundamentales:

- ..Al incrementar la producción nacional, de forma directa, se podría incidir en una sustitución inteligente de una serie de productos importados."

- " 'La cantidad adicional de bienes fabricados podría, también, ser orientada a la exportación en mercados de otros de países." (Araque J., 2012)

Son incontables los desafíos a los que se enfrentan las pymes, hacerles frentes y salir victoriosas es una tarea difícil si no se instruyen; es en este punto donde la academia puede accionar mediante proyectos de capacitación, asesoría y mejora, en especial en las actividades productivas que al país le interesa desarrollar. (Araque J., 2012)

\section{Uso de herramientas cuantitativas y cualitativas para la mejora en la gestión empresarial.}

La gestión empresarial se sustenta en los conocimientos que se tengan sobre: riesgo, beneficio y mercado. A diario los administradores de las pymes se enfrentan a desafíos, los cuales tienen que asumir y tomar decisiones. Las acciones que derivan de estas decisiones tienen 
consecuencias, positivas o negativas, en sus finanzas, en la relación con sus usuarios y en la evolución de su empresa, ya sean a corto, mediano o largo plazo. Tomar decisiones de forma empírica sin un análisis adecuado no es una buena estrategia, es probable que alguna sea efectiva, pero a la larga la estabilidad de la empresa menguará y su fracaso será casi seguro. No hay un modelo o estándar preestablecido que se pueda seguir siempre para garantizar el éxito al tomar decisiones, pero si existen herramientas para analizar y procesar los datos e información disponible las cuales aportan solidez a este proceso y minimizan los efectos colaterales negativos. (Velasco, 2010)

Una particularidad de las pymes es que son administradas frecuentemente por individuos con lazos filiares o de afecto cercanos, donde se asignan las responsabilidades gerenciales en función de estos y no en las habilidades de la persona, lo que limita la capacidad de dirección y gestión de estas empresas, y por ende su desarrollo. Como filosofía común suelen orientarse a crecer, lo que responde en primera instancia a sus intereses personales, y no en desarrollarse como organizaciones.

Dentro de los problemas más serios que afectan a las pymes se destacan los siguientes:

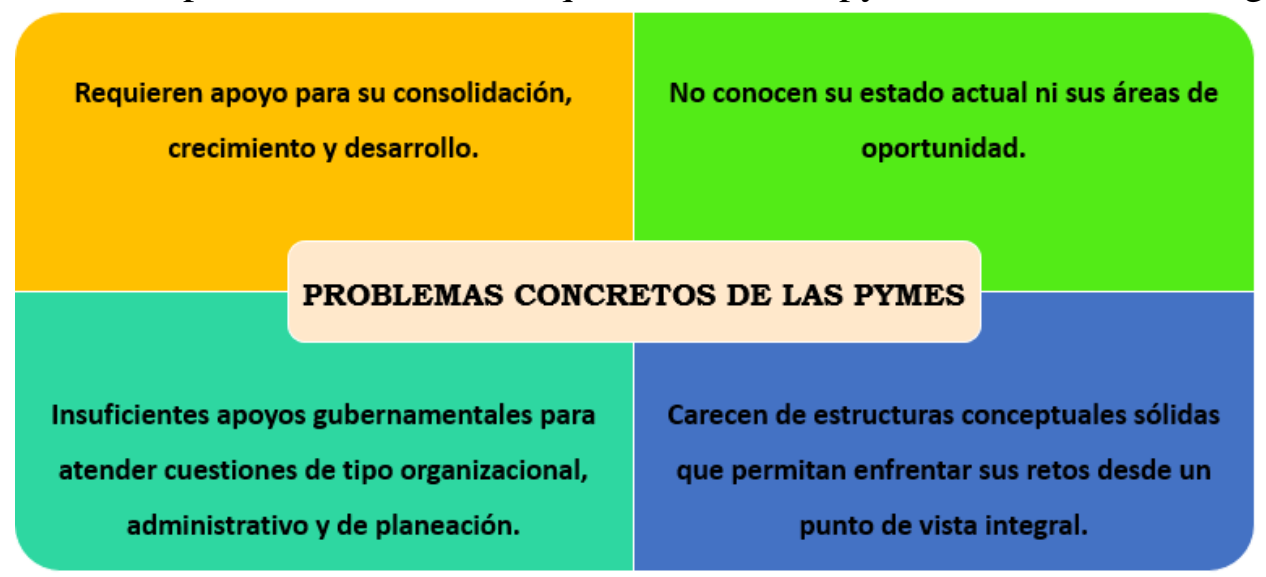

Figura 9. Problemas que afectan las pymes. Adaptado de: (Ordóñez Islas, 2012)

Para darle solución a los problemas relacionados en la Figura 7 y a otros mencionados previamente, los cuales pueden están indistintamente afectando a cada pyme de forma independiente, se requieren definir procesos de intervención que respondan a las necesidades del entorno en que está enmarcada en: tiempo, espacio y circunstancias. Estos procesos deben estar soportados por argumentos fuertes, criterios sólidos y la participación colectiva de todos los miembros de la organización. Implicar a todos los segmentos de la entidad, independientemente de su nivel jerárquico, y la retroalimentación pertinente son elementos que agregan valor al proceso. (Ordóñez Islas, 2012) propone un gráfico, en el cual representaba los resultados de dicho proceso de intervención en el eje de las ordenadas y el horizonte temporal en el eje de las abscisas. 


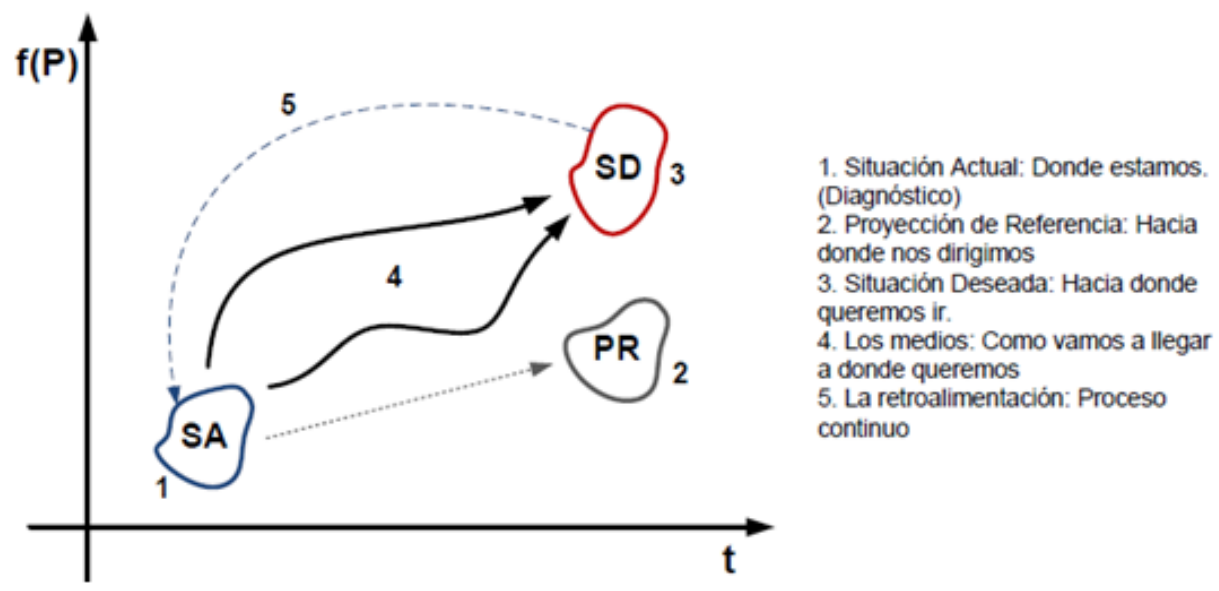

Figura 10. Función de los procesos de intervención.

Tomado de: (Ordóñez Islas, 2012)

La situación actual es el momento inicial. La proyección de referencia es el lugar al que por la inercia misma y sin llevar a cabo ninguna acción, es posible llegar. La situación deseada es el estado al que se quiere aproximar y los medios son la manera como se pretende lograrlo. La retroalimentación es un punto fundamental, porque representa la instancia de la evaluación y control, y permite otorgarle el adjetivo de continuo al proceso de intervención. Estas intervenciones deben ser un proceso continuo de aprendizaje y mejora. (Ordóñez Islas, 2012)

Por otra parte (Navarrete Báez, 2013) realizó un estudio multivariante para analizar las variables que influyen positivamente en los procesos decisionales de las pymes, resultando las más significativas las siguientes:

a. Contar con una visión clara de la empresa, donde todos sus trabajadores la comprendan y la sigan.

b. Mantener manuales de operación interno.

c. Tener un organigrama estructurado y comprendido por todos los empleados.

d. Poseer un catálogo de productos claro y oportuno de lo que les pueden ofrecer a sus clientes. (Navarrete Báez, 2013)

Lo perturbarte es que un porciento significativo de las pymes no tienen desarrolladas estas condiciones, por lo que son limitantes para su desarrollo.

La toma de decisiones no es siempre una tarea sencilla, pero ésta puede agilizarse, y mucho, si se hace uso de las herramientas para la mejora de la gestión.

La aplicación correcta de estas técnicas "conllevan significativas mejoras para los negocios derivadas de una mejor gestión de la información, un aumento de la productividad y de la gestión del equipo humano, una optimización del servicio al cliente con el consiguiente impacto en las ventas, una reducción en los costes y una mejora general de todos los procesos“. (Herranz, 2013) 
Alcanzar y mantener una vigilancia de la realidad del negocio es importante pues no se puede manejar toda la información disponible; seleccionar y procesar los datos adecuados en cada momento minimiza los errores que pueden estar dados por la percepción individual del administrador, la cual puede estar sesgada por su juicio personal. (Duff \& Media, 2016)

La aplicación de herramientas y técnicas cualitativas y cuantitativas debe integrarse en toda la gestión de la organización de las pymes para alcanzar estrategias proactivas en lugar de reactivas. Su uso "es de vital importancia para la optimización de los recursos y el desempeño de la empresa, tanto en lo administrativo como en la operación; beneficiando así desde los dueños hasta el propio cliente". (Aguirre Choix \& Armenta Velazquez, 2012)

No se trata de usarlas de forma desorganizada o por instinto, para darle solución a los problemas que las afectan a las empresas se requiere mantener un proceso estructurado y organizado para la mejora. En varios países de Latinoamérica se utiliza un proceso común para la garantía de la calidad, específicamente en centros de salud denominado " Proyecto de Garantía de Calidad", el cual combina métodos de gestión de la calidad que son usados estados desarrollados como: Estados Unidos, Europa, Canadá y Asia. Este se sustenta en un método cíclico e iterativo. (Ross, Zeballos, \& Infante, 2000)

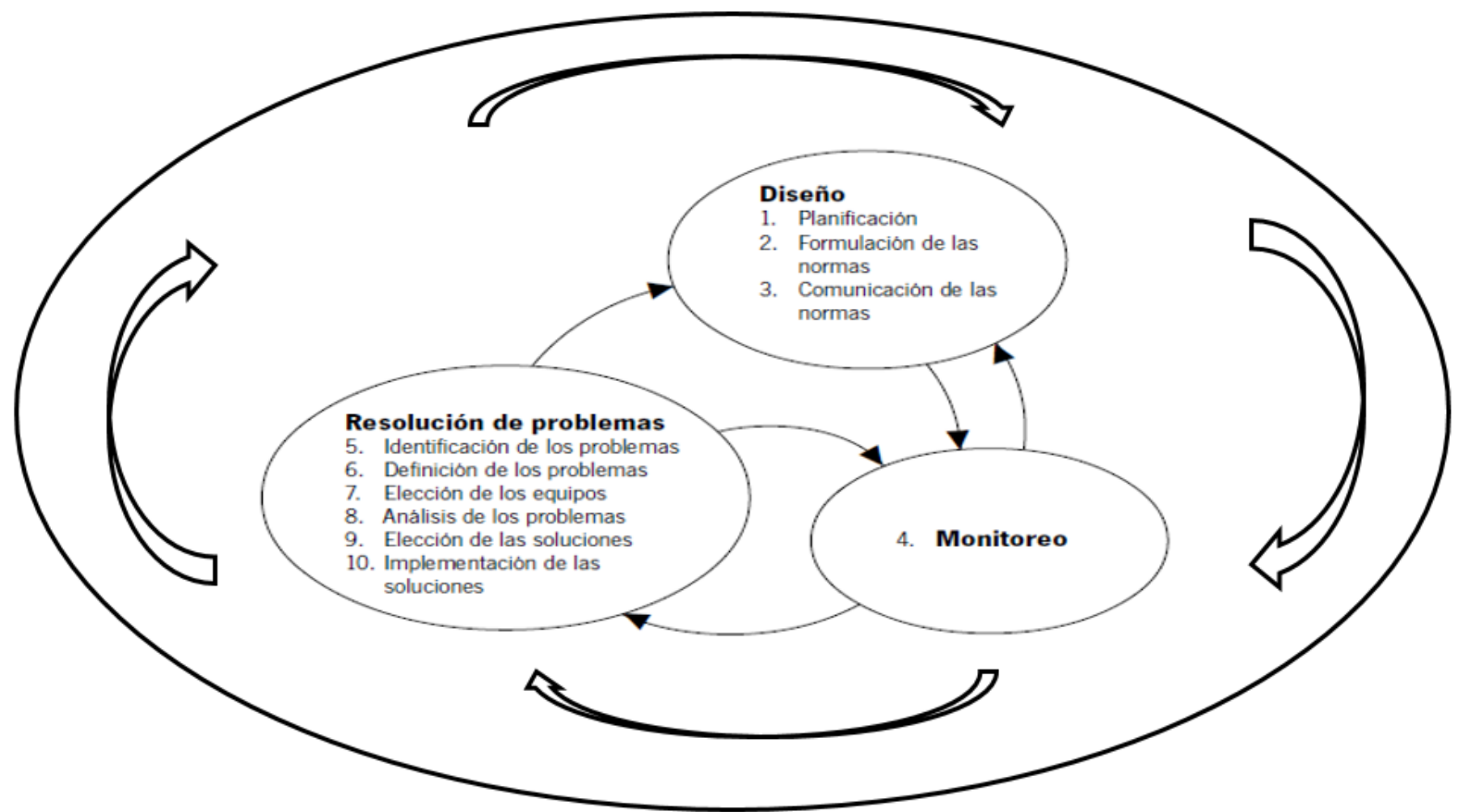

Figura 11. Metodología para los Proyectos de Garantía de Calidad. Adaptado de: (Miller Franco, Newman, Murphy, \& Mariani, 1997)

Al respecto (Miller Franco, Newman, Murphy, \& Mariani, 1997) en el método propuesto para la solución de problemas definen cuatro elementos básicos: a) "Concentración en las necesidades de los usuarios" (tanto los externos como los internos), b) "Concentración en los sistemas y procesos", bajo la premisa que "los problemas de calidad de un proceso se deben, con frecuencia, a la deficiencia de uno o más de los procesos afines del sistema o a una falla en la 
coordinación de los procesos relacionados entre sî", c) "Concentración en las decisiones que se basan en datos", al respecto los autores plantean que "el mejoramiento de los procesos requiere información con respecto a la forma en que funcionan, las decisiones que se tomen con respecto a aspectos problemáticos y mejoras tienen que basarse en los hechos, pero no en suposiciones.", d) "Concentración en la participación y el trabajo en equipo para mejorar la calidad", la efectividad en los procesos de mejora dependen en gran medida del nivel de involucramiento que tenga el personal, no solo los que estén desarrollándolo directamente sino todas las personas de la organización, en especial los que despliegan las actividades operativas al tener un conocimiento tácito de lo que puede estar fallando o puede ser perfeccionado, conocen mejor las causas; además al involucrársele se sienten parte de la solución y se motivan más al momento de aplicarla.

Un elemento que se debe destacar, es la importancia de los datos que se utilicen en dichos procesos, (Miller Franco, Newman, Murphy, \& Mariani, 1997) destacaban que en la medida de lo posible se debe verificar todo con datos. Durante todo el proceso de resolución de problemas se necesitan datos para 1) ayudar a detectar y definir problemas, 2) identificar las causas principales de los problemas y los procesos con errores, y 3) controlar los efectos de las soluciones implementadas para asegurarse de que funcionen.

Cómo premisas para alcanzar la efectividad deseada u esperada en las acciones que se deriven de los procesos de mejora deben cumplirse dos premisas básicas:

- Compromiso de la alta dirección y trabajadores de involucrarse en el proceso de toma de decisiones, haciendo uso de herramientas cuantitativas para contribuir a solucionar los problemas que se presenten.

- Aseguramiento de la asignación de los recursos necesarios para el desarrollo de los proyectos de mejora. (Garza Ríos, González Sánchez, Pérez Vergara, Martínez Delgado, \& Sanler Cruz, 2012)

Los procesos de mejoras deben estar sustentados en seis principios básicos:

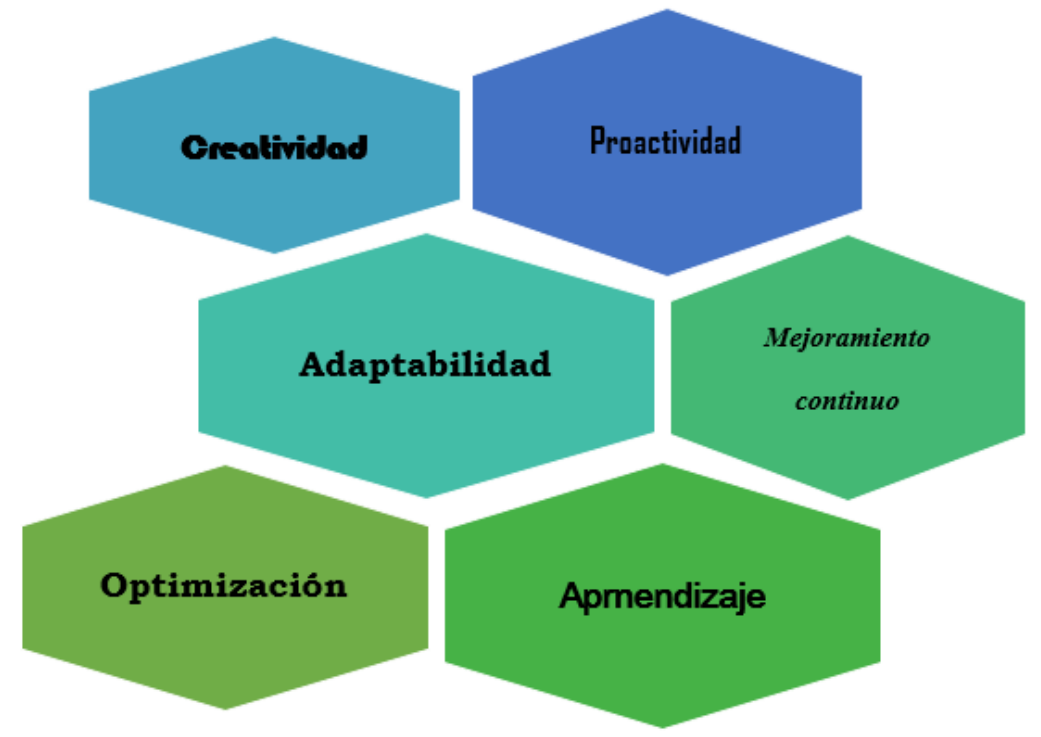

Figura 12. Principios básicos para la mejora 
Adaptado de: (Garza Ríos, González Sánchez, Pérez Vergara, Martínez Delgado, \& Sanler Cruz, 2012)_(García Caraballo \& Macías Mesa, 2010)

Según (Porter, 2003), "las empresas pueden ser más productivas en un sector si emplean métodos especializados y tecnología avanzada"

La toma de decisiones no es siempre una tarea sencilla, pero ésta puede agilizarse, y mucho, si se hace uso de las herramientas para la mejora de la gestión, para ello se proponen en la siguiente tabla un conjunto de técnicas y herramientas cualitativas y cuantitativas que pueden ser aplicadas indistintamente en las organizaciones, dependiendo del problema que se esté analizando y los datos e información con que se cuente.

Tabla 1: Herramientas de apoyo a la toma de decisiones para la mejora de la gestión empresarial.

\begin{tabular}{|c|c|}
\hline \multicolumn{2}{|c|}{ TÉCNICA O HERRAMIENTA } \\
\hline Gestión y Administración de Proyectos & $\begin{array}{l}\text { Gráfico Gantt } \\
\text { CPM } \\
\text { PERT } \\
\text { Método del Camino Crítico (CPM/PERT) }\end{array}$ \\
\hline $\begin{array}{l}\text { Entrevista } \\
\text { Análisis FODA } \\
\text { Dinámicas de grupo } \\
\text { Tormenta de Idea } \\
\text { Método Delphy }\end{array}$ & \\
\hline Hoja de datos & \\
\hline $\begin{array}{l}\text { Observación directa } \\
\text { Diagrama de Flujo } \\
\text { Diagrama de Causa-Efecto } \\
\text { Histogramas } \\
\text { Diagrama de Pareto } \\
\text { Diagrama de Dispersión }\end{array}$ & \\
\hline Control estadístico del proceso & $\begin{array}{l}\text { Gráficos de control por variables } \\
\text { Gráficos de control por atributos } \\
\text { Capacidad de los procesos }\end{array}$ \\
\hline $\begin{array}{l}\text { Diagrama de afinidad } \\
\text { Diagrama de relación } \\
\text { Diagrama de árbol }\end{array}$ & \\
\hline Diagrama de Matriz & \\
\hline $\begin{array}{l}\text { Benchmarking } \\
\text { Programación lineal } \\
\text { Problema de asignación } \\
\text { Problema de transporte } \\
\text { Problemas de Localización de Servicios } \\
\text { Control de inventarios } \\
\text { Técnicas multicriterios por atributos ( } \\
\text { Técnicas multicriterios por multiobjetiv }\end{array}$ & $\begin{array}{l}\text { DM) } \\
\text { ( MODM ). }\end{array}$ \\
\hline
\end{tabular}




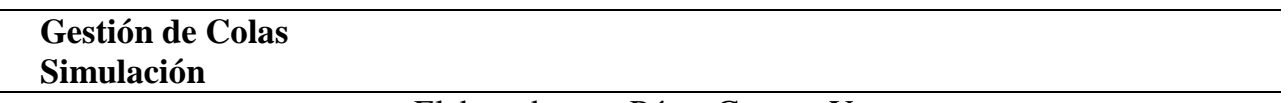

Elaborado por: Pérez Guerra, Y.

\section{Discusión}

Una intervención organizacional exitosa ayuda a lograr el desarrollo de una organización. Esto se representará directamente en un mayor número de empresas consolidadas (incremento en las unidades económicas del país), por lo tanto, mayor oportunidad de empleo y mayor producción a nivel nacional. De igual modo, independientemente del crecimiento de las unidades económicas, el desarrollo de una empresa incrementa su capacidad de producción. Con base en lo anterior y debido a la influencia que tienen las pymes en el país, los esfuerzos individuales por consolidar y desarrollar cada una de ellas, sumados a lo largo del tiempo, propiciarán beneficios importantes para éste.

Un proceso de toma de decisiones bien fundamentado desembocará en cambios y mejoras para las empresas, las cuales obtendrán como resultado su consolidación, crecimiento y desarrollo. Todo esto traerá consigo interesantes repercusiones positivas a nivel personal, empresarial y en el ámbito nacional.

El estudio de casos en empresas que sustentan sus procesos decisionales en herramientas sólidas muestra que la utilización este tipo instrumentos permite a las organizaciones elevar sus niveles de desempeño.

\section{Conclusiones}

Las principales conclusiones de la investigación son:

- Las pymes representan un segmento fuerte dentro del engranaje empresarial, económico y social en Ecuador, por lo que se requiere propiciar su desarrollo, lo cual puede lograrse a partir de estrategias de mejoramiento en su gestión.

- Los procesos decisionales son determinantes en el éxito o fracaso de cualquier entidad empresarial por lo que es necesario dejar de un lado la improvisación y establecer estrategias adecuadas para lograr su efectividad.

- La estrategia a utilizar en los procesos de toma de decisiones, así como las herramientas en las que se sustente, deben ser seleccionados por cada empresa dependiendo de sus condiciones momentáneas específicas (problemas u oportunidades de mejora) y sus características individuales.

- La utilización de técnicas cualitativas y cuantitativas como apoyo a los procesos decisionales dentro de la gestión empresarial conlleva a progresos significativos en: la gestión de la información, aumento de la productividad, gestión del capital humano, mejora del servicio al cliente, incremento en las ventas, reducción en los costes, en general, un perfeccionamiento en las organizaciones. 


\section{Bibliografía}

Aguirre Choix, R., \& Armenta Velazquez, C. E. (2012). La importancia del control interno en las pequeñas empresas en México. El Buzón de Pacioli, Año XII Número 76 Enero-Marzo , $1-17$.

Albanese, D., \& Boland, L. (2004). PyMes: algunas herramientas para diagnosticar su problemática. Escritos Contables. n.45 Bahía Blanca , 49-62.

Amaro Zárate, B. I. (2003). Valor Económico Agregado como estrategia financiera en las PYMES del sector automotriz. Puebla: Universidad de las Américas Puebla.

Andersen , A. (1999). Diccionario de Economía y Negocios. España: Espasa.

Araque J., W. (2012). Las PyME y su situación actual. Quito.: Observatorio PYME. Universidad Andina Simón Bolívar.

Bueno Campos, E. (2007). Organizacion de empresas: estructura, procesos y modelos (2a ED.) . Madrid: Piramide.

Duff, V., \& Media, D. (2016). Toma de decisiones gerenciales. La Voz de Houston and the Houston Chronicle, págs. http://pyme.lavoztx.com/toma-de-decisiones-gerenciales4091.html

Fundéu-BBVA. (2011). Fundéu-BBVA. Recuperado el 22 de Junio de 2016, de http://www.fundeu.es/recomendacion/pyme-no-pyme-ni-pyme-1049/

García Caraballo, J., \& Macías Mesa, J. A. (2010). El Proceso de Inteligencia Empresarial en las Empresas del Grupo de Diseño e Ingeniería de la. Revista de Arquitectura e Ingeniería, vol. 4, núm. 2, Versión On-line, disponible en: http://www.redalyc.org:9081/home.oa?cid=444279

García Del Junco, J., \& Casanueva Rocha, C. (2001). Prácticas de la gestion empresarial. Madrid: Mc Graw Hill.

Garza Ríos, R., González Sánchez, C., Pérez Vergara, I., Martínez Delgado, E., \& Sanler Cruz, M. (2012). Concepción de un procedimiento utilizando herramientas cuantitativas para mejorar el desempeño empresarial. Ingeniería Industrial. Vol.33 no.3, versión On-line ISSN 1815-5936.

González Jaramillo, R. (2013). Las MIPYMES ecuatorianas dinamizan la economía nacional. En H. J. King, Estudios industriales de la micro, pequeña y mediana empresa (págs. 16 - 18). Quito: FLACSO. 
Herranz, A. (12 de Septiembre de 2013). Cómo mejorar la toma de decisiones en la pyme. Recuperado el 22 de Junio de 2016, de http://www.ticbeat.com/pymes/como-mejorar-latoma-de-decisiones-en-la-pyme/

INEC. (2010). Censo Nacional Económico . Quito: Instituto Nacional de Estadísticas y Censos .

Instituto Nacional de Estadísticas y Censos. (2014). Directorio de Empresas y Establecimientos (DIEE). Quito: INEC.

Lemes Batista, A., \& Machado Hernández, T. (2007). Las pymes y su espacio en la economía Latinoamericana. Segundo Encuentro Internacional sobre las Medianas, Pequeñas y MicroEmpresas del Siglo XXI. On-line: Eumed.net.

Méndez Morales , J. S. (1996). Economía y la Empresa. México: McGraw-Hill.

Miller Franco, L., Newman, J., Murphy, G., \& Mariani, E. (1997). La resolución de problemas y el mejoramiento de procesos como medios para lograr calidad. Wisconsin : Center for Human Services.

Navarrete Báez, F. E. (2013). Las Micro, Pequeñas y Medianas Empresas de a Zona Metropolitana de Guadalajara: una perspectiva hacia la gestión de su proceso de toma de decisiones. Revista Electrónica Nova Scientia.Vol 5, num 10, 210 - 236.

Ordóñez Islas, P. A. (2012). Metodología para el diagnóstico organizacional de PYMES (Tesis). México, D. F.: Universidad Nacional Autónoma De México.

Pérez Gorostegui, E. (1994). Economía de la Empresa Aplicada. Madrid: Pirámide.

Porter, M. (2003). Ser competitivo: nuevas aportaciones y conclusiones. Barcelona: DEUSTO S.A.

Rodríguez Valencia, J. ( 2002). Administración de pequeñas y medianas empresas, 5ta Edición. México: Thomson.

Romero, R. (1999). Marketing. Palmir E.I.R.L.

Ross, A. G., Zeballos, J. L., \& Infante, A. (2000). La calidad y la reforma del sector de la salud en América Latina y el Caribe. Revista Panamericana de Salud Pública.vol.8 n.1-2, On-line version ISSN 1680-5348.

Secretaría Nacional de Planificación y Desarrollo. (2012). Plan Nacional de Desarrollo, Plan Nacional para el Buen Vivir 2009-2013: Construyendo un Estado Plurinacional e Intercultural. . Quito: SENPLADES.

Universidad San Martín de Porres. (2012). Organización y gestión de PYMES. Perú: Universidad San Martín de Porres. Facultad de Ciencias Administrativas y Recursos Humanos. 
Velasco, C. (9 de Febrero de 2010). La toma de decisiones del emprendedor a cargo de una Pyme. Coyuntura Económica, págs. http://coyunturaeconomica.com/emprendimiento/toma-dedecisiones-pyme. 